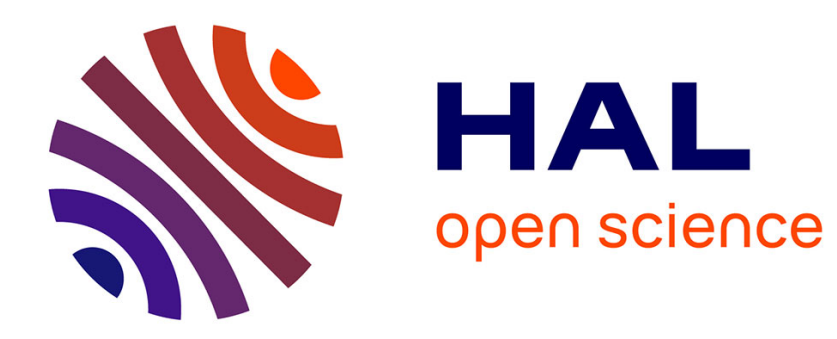

\title{
L'épithaphe d'Henri II Plantagenêt à Fontevraud
}

Robert Favreau

\section{To cite this version:}

Robert Favreau. L'épithaphe d'Henri II Plantagenêt à Fontevraud. Cahiers de Civilisation Médiévale, 2007, 50 (137), pp.3-10. 10.3406/ccmed.2007.2950 . halshs-03206686

\section{HAL Id: halshs-03206686 \\ https://shs.hal.science/halshs-03206686}

Submitted on 23 Apr 2021

HAL is a multi-disciplinary open access archive for the deposit and dissemination of scientific research documents, whether they are published or not. The documents may come from teaching and research institutions in France or abroad, or from public or private research centers.
L'archive ouverte pluridisciplinaire HAL, est destinée au dépôt et à la diffusion de documents scientifiques de niveau recherche, publiés ou non, émanant des établissements d'enseignement et de recherche français ou étrangers, des laboratoires publics ou privés. 


\section{L'épithaphe d'Henri II Plantagenêt à Fontevraud}

\section{Robert Favreau}

\section{Abstract}

Three English chroniclers have passed on the epitaph of Henry Plantagenêt's tomb at Fontevraud. Half the epitaph recalls the glory of the king, new Alexander ; the other half reminds the dead that happens unexpectedly ; and it invites to do good during our life. These verses give an account of the impact of a suddenly interrupted career that was exceptionally brilliant.

\section{Résumé}

Trois chroniqueurs anglais nous ont transmis l'épitaphe qui ornait le tombeau d'Henri Plantagenêt à Fontevraud. La moitié de l'épitaphe rappelle la gloire du roi, nouvel Alexandre, l'autre moitié évoque la mort, qui survient à l'improviste ; et elle invite à faire du bien pendant sa vie. Ces vers témoignent du retentissement qu'a dû avoir la brutale interruption d'une carrière exceptionnellement brillante.

\section{Citer ce document / Cite this document :}

Favreau Robert. L'épithaphe d'Henri II Plantagenêt à Fontevraud. In: Cahiers de civilisation médiévale, 50e année (n¹97), Janvier-mars 2007. pp. 3-10;

doi : https://doi.org/10.3406/ccmed.2007.2950

https://www.persee.fr/doc/ccmed_0007-9731_2007_num_50_197_2950

Fichier pdf généré le 25/03/2019 


\title{
Robert FAVREAU
}

\section{L'épitaphe d'Henri II Plantagenêt à Fontevraud}

\begin{abstract}
RÉSLIMÉ
Trois chroniqueurs anglais nous ont transmis l'épitaphe qui ornait le tombeau d'Henri Plantagenêt à Fontevraud. La moitié de l’épitaphe rappelle la gloire du roi. nouvel Alexandre. l'autre moitié évoque la mort. qui survient à limproviste : et elle invite à faire du bien pendant sa vie. Ces vers témoignent du retentissement qu'a dû avoir la brutale interruption d'une carrière exceptionnellement brillante.
\end{abstract}

\section{ABSTRAC"I}

Three English chroniclers have passed on the epitaph of Henry Plantagenêt's tomb at Fontevraud. Half the epitaph recalls the glory of the king. new Alexander: the other half reminds the dead that happens unexpectedly: and it invites to do good during our life. These verses give an account of the impact of a suddenly interrupted career that was exceptionally brilliant.

On peut penser qu'Henri Plantagenêt aura médité un jour sur l'épitaphe de Guillaume le Conquérant à Saint-Étienne de Caen, "grand roi gisant en une petite demeure ", ou sur celle de la reine Mathilde, à la Trinité de Caen, dite petite-fille, sœur et femme de roi. On est assuré qu'il connaissait fort bien le superbe mausolée réservé à la cathédrale du Mans à son père. Geoffroi Plantagenêt, mort à Château-du-Loir en $1151^{1}$. C"était déjà un insigne privilège que d'avoir enterré le comte à l'intérieur des murs de la cité, ce qui ne s'était pas encore fait². Le mausolée lui-même était dû à l'évêque Guillaume de Passavant, qui termina la reconstruction de la cathédrale consacrée en 1158. Il aura voulu célébrer la mémoire du comte qui avait ramené la paix en son domaine. et qui avait assuré aux évêques une bonne transmission de leur temporel. jusque-là trop souvent troublé lors des vacances du siège. Il établit un chapelain à l'autel du Crucifix sis près du tombeau. pour que le sacrifice soit offert chaque jour pour le comte. La plaque de cuivre émaillée qui ornait le tombeau. portant l'effigie de Geoffroi, est la "plus grande plaque de parement jamais produite par les émailleurs médiévaux »'. En 1712 elle est dite appliquée à un des piliers de la nef de la cathédrale. du côté nord. près de la chapelle du Crucifix qui servait alors d'église paroissiale-. Elle disparaît en 1792. se trouve en 1816 aux mains d'un collectionneur privé. à qui le département de la Sarthe l'achète à l'intention du Musée de la ville, où

1. "Chronica de gestis consulum Andegavorum". dans Chromiques des comtes d'Anjou, éd. Paul Marcht: (iay et André SAl.MoN. Paris. 1856. p. 156-157 et p. 293.

2. Chronique de Robert de Torignu, abbé du Momt-Saint-Michel, éd. Iéopold Di:1.1s1.1. Rouen. 1872 (Société de l'histoire de Normandie), t. I. p. 256.

3. Marie-Madeleine (jal ThHer. Émomx du moven age occidental. 2 éd.. Fribourg. 1972. p. 81-82. ill. p. 83. catalogue p. 327. n 4). La plaque mesure $6.3 \times 33 \mathrm{~cm}$.

4. Histoire littéraire de la France. t. 9. par Paulin Par1s. Paris. 1868. p. 165. 
on peut aujourd'hui laadmirer. L'inscription, distique élégiaque avec rimes entre les hémistiches et entre les fins de vers, fait l'éloge du pacificateur et du protecteur des églises :

\section{ENSE TUO. PRINCEPS. PREDONUM TURBA FUGATUR ECCLESIISQUE QUIES, PACE VIGENTE. DATUR.}

Par ton épée. prince. la foule des pillards est mise en fuite.

et. grâce à la paix qui règne. la tranquillité est assuréc aux églisess.

Après avoir dit que Geoffroi fut inhumé en la cathédrale. devant le Crucifix, Robert de Torigni. abbé du Mont-Saint-Michel, donne un distique qui a peut-être été inscrit sur le tombeau :

Huic Detus aeternum tribuat conscendere regnum

Quatinus angelicis turmis conregnet in aevum

Que Dieu lui accorde daccéder au royaume éternel

Où il règne pour toujours avec les troupes des anges.

Ces deux vers terminent l'épitaphe de l'abbé du Bec-Hellouin. Guillaume, mort en 1124. rapportée par le même Robert de Torigni". Henri Plantagenêt s'est associé à l'honneur rendu à son père en faisant don à la cathédrale du Mans, en 1161. d'une rente de quarante livres pour que deux prêtres assurent quotidiennement, pour le salut de l'âme de son père. le service de l'autel placé devant le tombeau?

Une intervention d'Henri II en Berry, peu avant cette date. montre qu'il avait pleinement conscience de l'intérêt d'une inscription pour affirmer publiquement une politique. Ebbes II. seigneur de Déols, disputait à Garnier de Clins-Dessus le titre de fondateur et la fonction de gardien (custos) de l'abbaye de Varennes, et. pour que ce droit lui soit pour toujours reconnu, il avait fait placer dans l'abbaye une dalle portant gravés les titres qu'il revendiquait. Henri II obligea Ebbes II à faire enlever la dalle, parce quill voulait être considéré lui-même comme "le fondateur, gardien et défenseur de ladite église " ${ }^{\star}$. On peut citer, dans le même ordre, l'inscription qui disait Ebbes V. seigneur de Charenton : le texte en sera altéré par les seigneurs de La Châtre qui se prétendaient les fondateurs de l'abbaye".

Henri Il avait reçu une excellente éducation par le grammairien maître Pierre de Saintes puis, surtout, à la cour de son oncle, le très cultivé comte de Gloucester, protecteur de Geoffroi de Monmouth. On connaît la générosité de son mécénat, son intérêt marqué pour le patronage littéraire"'. lui que Benoît de Sainte-Maure loue comme le souverain :

qui meuz connoist ovre bien dite

e bien seant e bien escrite.

5. M.-M. GAl"THIt:R (op. cit. n. 3). p. 82: - EAD.. "Art. savoir-faire médiéval et laboratoire moderne. à propos de leffigie funéraire de (ieoffroy Plantagenêt ". Comptes rendus de l'Académie des inscriptions et belles-lettres, 1979. p. 105131: - Marcel Dirras. Maine roman. La Pierre-qui-Vire. 1985 (Zodiaque. La nuit des temps. 64). p. 215: - ID). Anjou roman. 2 éd. La Pierre-qui-Vire. 1987 (Zodiaque. La nuil des temps. 9). p. 21.

6. Chronique de Robern de Torigini (op. cit. n. 2). p. $168-169$ et 256 : - Corpus des inscriptions de la France médiévale, 1. 22. éd. Robert Favri:Al; el † Jean Mi(halid). Paris. 2002. p. 121-122.

7. Recueil des actes de Henri II roi d'Angleterre et duc de Normandie concernant les provinces françaises et les affaires de France, éd. Léopold Dl:I.ISLE el Élie BER(iER. T. I. Paris. 1916. n 70) p. 172-173.

8. Ibid., n 124 bis. p 230-231. (hinon, 1159?: - Eugène HIBb:RT, Cartulaire des seigneurs de Châtecutroux. Châteauroux. 1931. donne la date de 1155: - Guy Divinutuy. Le Berry du x" siecle au milieu du xurt. Étude politique. religieuse, sociale et écomomique. Paris, 1973, p. 405-406. Je remercie mon collègue Martin Aurell de mavoir signalé ce précieux témoignage épigraphique.

9. F. DI:SHot:II:RI:S. "Monuments funéraires relevés en Berry par dom ("latde Fstiennot. Notes et commentaires". Memoires de la Sociéte des Antiquaires da Centre, 40. 1921. p. 186-187.

10. Walter F: SCHIR.MER et Ulrich BROICH, Studien zam literarischen Patronat im England der 12. Jahrhunderts. Cologne/Opladen. 1962 (Wisienschaftliche Abhandlungen der Arbeitsgemeinsclaft für Forschung des Landes NordrheinWestfalen. 23). 
Une thèse récente a mis en valeur L'idéologie Plantagenêt ${ }^{11}$, et en particulier la volonté de rattacher l'histoire des Plantagenêt à celle du roi Arthur, le preux conquérant et sage primus inter pares des chevaliers de la Table ronde. "union de la bravoure et de la sagesse à la tête de l'État". Henri II. "nouvel Arthur", va "incarner l'alliance de la clergie et de la chevalerie qui s'accomplissent en sa personne». C'est Geoffroi de Monmouth qui le premier a mis en forme l'histoire légendaire du roi Arthur dans son Histoire des rois de Bretagne qui connaîtra un succès prodigieux dont témoigne le nombre exceptionnellement élevé des manuscrits conservés. Henri et, après lui, Richard, s'annexeront ainsi la matière de Bretagne, en contrepoint du succès du cycle de Charlemagne auprès des Capétiens.

Mais Henri II a été aussi comparé à Alexandre le Grand. Un anonyme poitevin traduit à cette époque. pour le public anglo-normand, la version franco-provençale du Roman d'Alexandre, et Lambert le Tort donne une continuation à cette nouvelle branche vers 1170). Un peu plus tard. Thomas de Kent écrit une geste complète d'Alexandre dans son roman Roman de toute Chevalerie (vers 1175). Jean de Salisbury, dans son Policraticus, compare Henri II à Alexandre le Grand pour sa fougue. sa jeunesse, ses victoires. Dans le dialogue qu’Étienne de Rouen imagine, dans son Draco Normannicus, entre le roi Arthur et Henri II, le premier se dit "tels Alexandre le Grand et César ", le second lui répond en se comparant au même modèle d'Alexandre le Grand". Dans une lettre à Henri II, à propos de l'éducation de son fils Henri le Jeune. Pierre de Blois écrit qu" "un roi sans lettres est un bateau sans rames et un oiseau sans plumes » et il cite "Jules César. maître ès littérature, droit civil et philosophie. Alexandre, formé par Aristote ainsi que les rois instruits de l'Ancien Testament (Salomon, David, Ézéchias, Josas) ou de l'Empire romain chrétien (Constantin. Théodose. Justinien, Léon) aussi versés dans l'art militaire que dans les lettres ${ }^{\prime 1}$. Giraud de Barri, chapelain du roi (1184), envoyé en Irlande après la prise de possession de l'île nouvellement conquise (1185). termine en 1187 sa Topographia Hibernica et la dédie à Henri II : «Vos victoires délient les bornes de la terre : vous, notre Alexandre d'Occident, avez étendu votre bras depuis les Pyrénées jusqu'aux confins occidentaux de l'Océan septentrional $»^{14}$. Giraud fut "probablement un témoin oculaire des derniers instants d'Henri II à Chinon "s.

Henri II aurait pu, comme d'autres grands lettrés, prévoir son épitaphe. mais il a été surpris par la mort, et après seulement quelques jours de fièvre, il est décédé à Chinon le 6 juillet 1189 . Son corps fut porté en l'abbaye proche de Fontevraud, où, bien qu'il eut été abandonné par ses enfants, il fut inhumé par les archevêques de Trêves et de Tours, "comme reis », "a plus bel service $k$ il pourent, si comme apparteneit a rei. selon Dieu e selon la lei " $^{\prime \prime}$. On avait revêtu le corps des vêtements de lin imprégnés de chrême de sa consécration royale ${ }^{17}$. il avait une couronne d'or sur la tête, des gants aux mains. des chaussures tissées d'or et des éperons aux pieds. un grand anneau au doigt, un sceptre à la main, un glaive au côté, le visage découvert ${ }^{1 \mathrm{x}}$. Le doyen de Londres. Raoul de Dicet et, après lui, de façon moins complète, Roger de Wendower et Matthieu Paris nous ont heureusement transmis le texte qui fut inscrit sur te tombeau du roi" :

\section{SUFFICIT HIC TUMULUS CUI NON SUFFECERAT ORBIS}

RES BREVIS EST AMPLA CUI FUIT AMPLA BREVIS

11. Amaury Cualot: Lüdéologie Plantagenêt én Occident (x/t-xV siécles), Rennes. Université de Rennes II. 2000): thèse publiée sous le titre : Lidéologie Plantagenêt. Royauté arthurienne et monarchie politique dans lespace Plantagenêt $(x\|/-x\| I r$ s.). Rennes. PUR. 20(0)] (Histoire).

12. Ibid. passim (index : Alexandre le (irand).

13. Martin Atri:1. L'Limpire des Planagenêt 1154-1224. Paris. 2003. p. 108.

14. Ibid., p. 9: - A. Chalol: (op. cit. n. 11) (thèse publ. 2001]. p. 73.

15. Ihid.. p. 74.

16. Lisistoire de Guillaume Le Maréchal. comte de Siriguil at de Pembroke, régent dangleterre de 1216 à 1219 . poème publié par Paul MEYı:R. 1. I. 1891. p. 331. 334. 340.

17. Chroniques des comtes dinjoit (op. cit. n. 1). p. 346.

18. Mathaei Parisiensis monachi Sancti Albani (hronica majora. II : AD. I067 to AD). 1216. éd. Henry Richard LI ARD). Londres. 1874 (Rerum Brittanicarum Vedii Aevi scriptores).

19. Ibid.. p. 345 (ne donne que six vers) : Radulfi de Diceto decani Londoniensi opera historica. éd. William Sn'BBS. II. Londres. 1876 (Rerum Britannicarum Medii Aevi scriptores). p. 65 (les dix vers): - Ro(il:R D): Wi:NoOW:R. The Flowers of History, éd. Henry G. HrwlıT, vol. I. Londres. 1886 (six vers). 


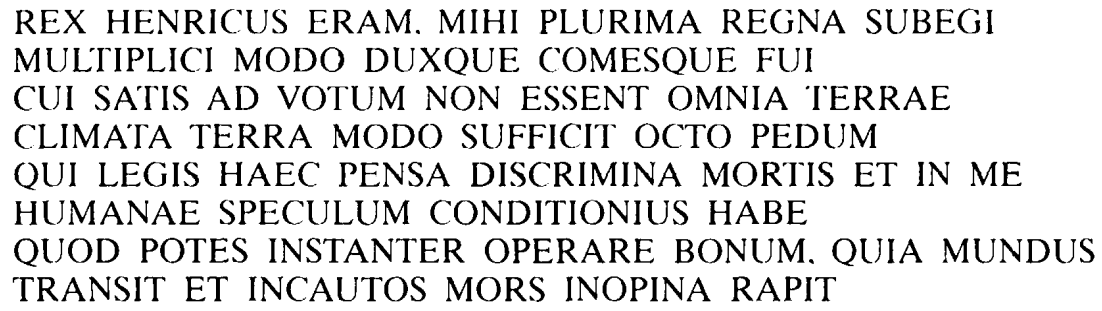

$\mathrm{Ce}$ tombeau suffit. à qui le monde n'avait pas suffi.

Ce lieu restreint est magnifique. pour celui à qui fut magnifique une vie brève.

J'étais le roi Henri. j’ai soumis à mon pouvoir un très grand nombre de royaumes.

De bien des manières, et je fus et duc et comte.

Lui à qui toutes les régions de la terre n’étaient pas assez à son souhait.

Une terre de seulement huit pieds suffit.

Toi qui lis, médites ces séparations de la mort. et en moi

Vois le miroir de la condition humaine.

Fais dans linstant le bien que tu peux. car le monde

Passe, et la mort. survenant à limproviste. enlève ceux qui ne sont pas sur leurs gardes.

L'épitaphe est composée de cinq distiques élégiaques. Elle ignore le vers léonin si habituel à cette époque, et paraît composée laborieusement. Le premier distique est bien tourné. mais c'est un emprunt. Le deuxième est le seul à faire intervenir le roi à la première personne. le pentamètre emploie trois fois le -que enclitique, ce qui est beaucoup. Dans les trois distiques suivants, le sens n'est pas complet vers par vers, mais chevauche la fin de l'hexamètre et le début du pentamètre. Les expressions métriques probablement empruntées y sont nombreuses, qui legis, discrimina mortis, mors inopina rapit. Quel est donc le message que l'on a voulu. maladroitement. transmettre?

Matthieu Paris précise qu'au cours de sa vie le roi Henri avait coutume de dire que le monde entier ne devrait pas suffire à l'ambition d'un prince. Et comme on lisait devant lui, alors qu'il attendait la mort, des vers sur "le tombeau qui désormais suffit à celui à qui le monde n'avait pas suffi », il aurait dit qu ils lui plaisaient bien et qu'il faudrait les inscrire sur sa tombe pour l'édification des générations futures. Son souhait fut exaucé. et ces vers formèrent le début de son épitaphe. Il s'agissait du distique de l'épitaphe d'Alexandre le Grand, que l'on trouve, avec des variantes dans divers manuscrits du Moyen Âge". L'Anthologie latine publiée par Alexander Riese donne pour deuxième vers : cui nimis angusta [est], fuit ampla nimis et. pour un des manuscrits, le texte cité dans l'épitaphe d'Henri. Dans un manuscrit de la Bodléienne (Digby 53), on trouve la variante Sufficit iste lapis: ${ }^{-1}$ et le distique figure aussi dans des manuscrits de Londres et d'Oxford du XII ${ }^{\mathrm{s}} \mathrm{s} .^{22}$. Ces vers devaient donc être bien connus dans l'entourage du roi Henri. Leur emploi pour le Plantagenêt revient à faire de celui-ci un nouvel Alexandre.

On observera qu'Henri II a autour de lui des lettrés, qui accompagnent ses dernières heures en lui lisant des vers, et parmi eux Giraud de Barri qui l'a donné "comme notre Alexandre d'Occident». Dans le Roman d'Alexandre le conquérant distribue son empire entre ses douze pairs et, après sa mort, suit la déploration de chacun d'entre eux. Mas 'Udi fait intervenir vingthuit sages après la mort d'Alexandre, et Pierre Alphonse vingt-deux philosophes.

Un Grec d'Alexandrie avait composé au II $^{*}$ s. une histoire fabuleuse d'Alexandre le Grand, qui fut traduite en latin au $I^{*}$ s. et résumée au IX $\mathrm{s}$. en un Epitome. C'est dans les États d'Henri II,

20. Antologia latina sive poesis latinae supplementum. Pars prior: Carmina in codicibus scripta. éd. Alexander RItSs:. Vol. 2 : Reliquorum librorum carmina. I.eipzig. 1870. p. XIVII. n. 26.

21. Paul Mryl:R. "Troisième rapport sur une mission littéraire en Angleterre el en Écosse". Archives des missions scientifiques et littéraires. Chotx de rapports et instructions. 2 sér. 5. 1868. p. 185. Le second vers est celui donné comme inscrit sur le tombeau de Fontevraud.

22. Hans WatTHE:R. Carmina medii aevi posterioris latina. $\mathrm{I} / 1$ : Initia carminum a versum medii aevi posterioris latinorum... 2 éd.. Göttingen. 1969, p. 980. n" 18695 et 18696. 
comme on l'a dit ci-dessus que prend forme le Roman d'Alexandre, en vingt mille vers de douze syllabes, d'où le nom d' "alexandrins" donné à ce type de vers.

L'épitaphe latine d'Alexandre est déjà connue au $X^{*} s^{3.3}$, car on en trouve un écho évident dans l'épitaphe du duc Liudolfe dc Saxc. fils d'Othon Irr :

\section{NON MIHI LIUDOLFO TOTUS SUFFECERAT ORBIS:}

La terre entière ne mavait pas suffi. à moi Liudolfe.

Le contexte en est donné dans l'ouvrage de Mas 'Udi, mort en 956. traduit sous le titre Les prairies d'or:. À la mort d'Alexandre. "les sages qui l'avaient accompagné. tant grecs que perses. indiens et autres de toutes les nations, et qu'il avait coutume de réunir et de consulter, ne décidant jamais rien sans prendre leur avis, se rassemblèrent autour de ses restes", et le premier d'entre eux demanda "que chacun prononce une parole qui soit une consolation pour les grands et un avertissement pour le peuple ». Le quatorzième dit : "Ô toi pour qui le monde était trop limité en longueur et en largeur, je voudrais bien savoir comment tu te trouves dans l'étroite prison qui t'enserre maintenant", et le vingt-troisième proposa : "Tu serais bien heureux de posséder un seul de tes membres, alors que la possession de toute la terre ne te suffisait pas ". Les dits des sages autour du corps d'Alexandre sont cités, au XII' s., par Pierre Alphonse dans sa Disciplina clericalis, fabula $X X X$ : Il est dit d'Alexandre que sa sépulture en or était placée à la vue de tous, et que plusieurs philosophes s'y rassemblèrent. L'un deux dit : "Alexandre s'est fait un trésor avec l'or, maintenant l'or fait de lui un trésor $»^{20}$. Un autre dit : " Hier le monde entier ne lui suffisait pas (heri totus ei non sufficiebat mundus), aujourd'hui quatre brasses seulement lui suffisent ", etc."?.

Le sufficit hic tumulus de l'épitaphe d'Henri II peut être rapproché du sufficit et magno parva domus domino de l'épitaphe de Guillaume le Conquérant ${ }^{2 \times}$ ou du cui sufficerat locus unus de l'épitaphe de Richard Cœur de Lion à Fontevraud"'. Mais seule l'épitaphe d'Henri II est un rappel direct d'Alexandre le Grand.

La traduction du deuxième vers est difficile. Le res brevis semble bien être le tombeau. que d'autres épitaphes disent urna brevis (Saint-Victor de Paris, 1141, abbaye de Lannoy à Roy-Boissy, 1166), brevis lapis (abbaye de Longpont, XIII' s.), brevis loculus (Limoges, XIII' ${ }^{\circ}$ s.). Le second brevis pourrait renvoyer à la brièveté d'une vie que rappellent de nombreux textes bibliques : "brefs sont les jours de l'homme" (Job 14, 5), "brève est cette vie " (Sagesse, 15, 9). "la vie brève " (Ecclésiastique 10,11) par exemple. On sait les auteurs d'inscriptions des $\mathrm{XI}^{\mathrm{*}}$ et $\mathrm{XII}^{\mathrm{*}} \mathrm{s}$. friands de ces jeux de mots.

Le deuxième distique de l'épitaphe d'Henri II rappelle les conquêtes du roi et ses nombreux titres. Les Chroniques des comtes d'Anjou nous disent qu'Henri avait condensé les titres hérités de ses prédécesseurs sous la forme : Henri, roi d'Angleterre, duc de Normandie et d'Aquitaine. comte d'Anjou i", ce qui correspond exactement à la pratique de la chancellerie anglaise. L'épi-

23. On trouvera un état des éditions et une importante bibliographic dans le Dictionnaire des lettres françaises. Le Moyen Áge. nouv. éd. dir. Genviève Haskionr et Michel ZiNk. Paris. 1992. p. 1306-1308 ("Roman d'Alexandre". notice de L.F.F. el (.R.).

24. Franz-Xaver Krats. Die christlichen Inschriften der Rheinlander von der mitte des achten bis zu mitte des dreizehnten

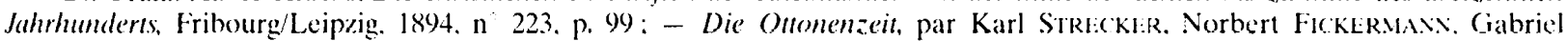
Sil.s(il. Bernhard Bisciloft. $1937 / 79$ (M.G.H.. Poetac latini aevi carolini). p. 321.

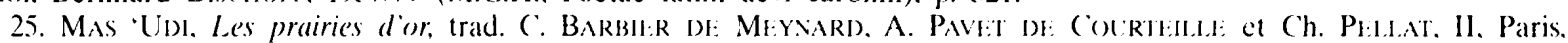
1965 . p. $252-254$.

26. Un des sages au dire de Mas 'Udi. aurait déclaré : "Cet Alexandre qui enfouissait lor. le voilà maintenant enfoui dans l'or"

27. P.L.. 1. 157. col. 705-706.

28. Corpus des inscriptions de la France médiévale (op. cit. n. 6). p. 46-47.

29. Antoine PI:RRIV:. "De nouvelles précisions sur la mort de Richard ('éur de Lion ". Bulletin de la Société archéo)logique et historique du Limousin. 87. 1958. p. 39.

30. II. rex Anglorum, et dux Vormannorum et Aquitanorum et comes Andegavorum : Chronique des comtes dAnjou (op. cit. n. 1). p. 342 
taphe se borne à le dire roi, duc et comte. Il n’y avait cité, bourg. ville. donjon. fort, château "dont li prince et li duc ne t'aient fait hommage" dit le Roman d'Alexandre.

Le troisième distique reprend, sous une forme bien moins élégante, le premier vers de l'épitaphe d'Alexandre et s'inspire des dits d'Alexandre, rappelés par Pierre Alphonse, sur l'étroitesse de l'espace désormais réservé à Henri II, comme cela fut le cas pour Alexandre. "Tiens or peu de terre " dit le Roman d'Alexandre.

Les quatre derniers vers constituent une apostrophe au lecteur pour l'inviter à méditer sur la mort à partir de l'exemple d'Henri II et à faire le bien sans remettre à plus tard, message banal dans l'épigraphie funéraire.

L'apostrophe au lecteur est très fréquente dans les épitaphes médiévales. Elle introduit le plus ordinairement une demande de prières, plus rarement des considérations sur les fins dernières et une exhortation morale. L'emploi de la deuxième personne du singulier - «toi qui lis » - et de l'impératif n'est pas fréquent, mais conduit à une interpellation plus vigoureuse et immédiate que la troisième personne. Le lecteur est invité à considérer le défunt roi comme un miroir de la condition humaine, ce qui en soi est une généralité fort banale, si ce n'est que le rang élevé du défunt, ses succès en ce monde rendent plus éloquente la leçon sur la vanité d'une brillante carrière face à la mort. L'expression discriminis mortis est employée par Paulin de Nole et par Aldhelm de Malmesbury, et se trouve dans une inscription carolingienne du trésor de la cathédrale de Bambergit.

Une bonne quinzaine d'inscriptions médiévales comportent le mot speculum, le Christ est dit "miroir du Père ", Marie. "miroir de charité, de justice, de sainteté, de vertu, des vierges ", et "les miroirs" fleuriront dans la littérature du XIII" s., à commencer par Vincent de Beauvais, mais je n’ai pas rencontré dans les inscriptions l'expression de "miroir de la condition humaine ». Cette leçon sur la condition humaine est évoquée, avec un vocabulaire proche (sufficit, brevis) dans l'épitaphe de l'empereur d'Allemagne Conrad III, mort le 15 février 1152 à Bamberg où il fut inhumé

\section{QUI VESTES GERITIS PRETIOSAS. QUI SINE FINE \\ NON PROFECTURAS ACCUMULATIS OPES. \\ DISCITE QUAM PAUCIS OPIBUS POSTE FUNERA SITIS \\ CONTENTI. SACCUS SUFFICIT ATQUE LAPIS \\ CONRADUS REX JACET. QUI TO'T CASIELLA. IOT' URBES \\ POSSEDIT. TUMULO CLAUDITUR ISTE BREVI: \\ Vous qui portez des vêtements précicux et qui sans fin accumulez des richesses qui ne vous profiteront pas. \\ sachez que vous vous satisferez de peu de biens après votre trépas : \\ un linceul et une pierre suffisent. \\ Le roi Conrad git ici. qui posséda tant de châteaux. tant de villes : \\ Il est enterré en cet étroit tombeau.}

La vanité d'une carrière exceptionnellement brillante stoppée net par la mort, c'est bien le sens des vers que Guillaume Le Breton a consacrés à la mort d'Henri II au livre III de la Philippide : "Qu'est-ce que la gloire du monde, le luxe, la richesse, le pouvoir suprême, pour celui auquel récemment châteaux et villes pouvaient à peine donner l'hospitalité, et qui ne lui suffisaient pas quand il les avait prises? Maintenant une fort étroite maison l'enferme tout entier... Égale pour

31. PAltIN DI: Nol.t. Poema XVI. P.L., 61. col. 482 (M.G.H., Auctores Antiquissimi, éd. R. Erwal.D), t. 15, p. 364):

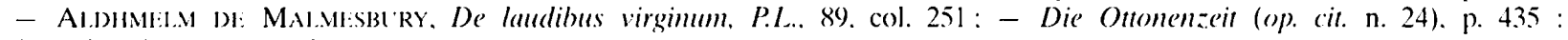
Ecce leo fortis transit discrimina mortis.

32. Jahrbïcher des Vereins von Alternumsfreuden im Rheinlande, 50/1. 1871, p. 202. Lépitaphe d'Hugues III archevêque de Rouen († 1164) parle aussi " des membres enfermés dans un étroit tombeau ", brevi clauduntur membra sepulcro (Corpus des inscriptions de la France médiévale, t. 22, Paris. 20(2) p. 288). 
tous. la mort termine impérieusement toute fortune ${ }^{3}$. Il est clair que la mort soudaine d'Henri II. au faîte d'une vie emplie de succès. mais aussi dans l'hostilité ou le quasi-abandon de ses enfants. aura frappé les esprits du temps.

L'épitaphe se termine par une exhortation à faire le bien sans remettre à plus tard. On peut rapprocher de l'épitaphe d'Henri II, le quod potes id tempta des Distiques de Caton (livre VI) ou le "évite le mal, fais le bien" (malum vites, bona fac) de la cathédrale Saint-Zénon de Pistoia $^{\text {is, }}$, mais la formule qui a eu la plus grande fortune dans les manuscrits ${ }^{35}$ et dans les inscriptions est :

\section{FAC BENE DUM VIVIS POST MORTEM VIVERE SI VIS}

Fais le bien pendant que tu vis. si tu veux vivre après la mort.

On la trouve dans les inscriptions funéraires à Lolme en Dordogne au XII" s. à Saint-Génis-desFontaines en 1271, à Saint-Bertrand-de-Comminges en 1317, et sur un chapiteau de Lazare et du mauvais riche au cloître de la cathédrale de Monreale à l'époque romane ${ }^{3 \hbar}$. L'óriginalité de l'épitaphe du Plantagenêt est d'insister sur l'instant, ce qui est plus pressant que l'invite à faire le bien pendant sa vie.

Le monde qui passe, la soudaineté de la mort, ces considérations évoquées à la fin de l'épitaphe d'Henri II n'ont rien d'original, et pourraient se trouver chez les philosophes autant que chez les moralistes chrétiens. Comme il s'agit d'une épitaphe du XII ${ }^{c}$ s., on en cherchera la source du côté chrétien. On trouve l'expression mundus transit dans la première lettre de saint Jean $(2,17)$, et saint Colomban $(\dagger 615)$ écrira : mundus enim transibit, et cottidie transit ${ }^{37}$, "le monde passera et il passe chaque jour». Plus proche d'Henri II, Serlon, abbé de l'Aumône entre 1171 et 1173 , a une étonnante composition métrique où il répète vingt et une fois l'expression mundus abit, «le monde s'en va »'. L'imprévu de la mort est martelé par le Christ dans l’Évangile de Matthieu aux chapitres 24 et 25 : "Vous ne savez ni le jour ni l'heure", et c'est la leçon des Vierges sages et des Vierges folles, à diverses reprises représentées dans l'art roman. L'expression même de "mort inopinée", mors inopina, se trouve dans le De sobrietate de Milon de Saint-Amand au $I X^{\prime}$ s. et dans les Poèmes de Baudri de Bourgueil au $\mathrm{XII}^{\circ}$ S."." Elle figure dans les épitaphes de l'abbé Guillaume à Trêves en $958^{411}$ et de Fulbert, évêque de Chartres, en $1029^{41}$. Quant au mors rapit, on le lit dans l'épitaphe de saint Adalhard, abbé de Corbie, à la cathédrale de Beauvais, vers 826, et de Delphie au musée de Cluny, fin $\mathrm{XI}^{*}$ - début $\mathrm{XII}^{\circ} \mathrm{s} .{ }^{12}$.

33. Euvres de Rigord et de Guillaume le Breton, historiens de Philippe Auguste, éd. H. François Dt:ABORDI: II : Philippide de Guillaume le Breton. Paris, 1885 (Société de l'histoire de France). p. 93-94. On retrouve des mots des épitaphes d'Henri II et de Conrad III.

34. Caloin B. KI:NDAl... The Allegory of the Church. Romanesque Portals and their Verse Inscriptions, Toronto/Buffalo/Londres. 1998. p. 25.5.

35. Hans Wattht: Carmina Medii Aaevi posterioris latina, I/1, Gottingen, 1969, n 6199, p. 312. et II/2. ihid.. 1964. n 86.35 . p. 3.

36. Pour Lolme, restitution du texte après envoi de photographies : pour Saint-Genis-des-Fontaines. Corpus des inscriptions de la France médiévale. éd. Robert Favri:st: Jean Michatd. Bernadette Mora. t. II. Paris. 1986. p. 144-145: pour Saint-Bertrand-de-Comminges, ibid.. 1. 8. 1982. p. 71: pour Monreale. Roberto SABATINi, Il chiostro di Momreale e la scultura romanica in Sicilia. Palerme. 1962. p. 92.

37. S. Col.ombas. Qualiter monachus Deo placere debet?. dans les Sancta Columbani opera. éd. WaI.Ki:R. Dublin. 1957. Instr. 111. 1.

38. Corpus des inscriptions de la france médiévale. 20 : Côte-dOr. éd. R. Falriall. J. Michatd. B. Mora. Paris. 1999. p. 1())-101.

39. Milon DI: SAINi-Andavd, De sobrietate, livre III, vers 433, dans Poetae latini aevi carolini, t. III. éd. Ludovicus

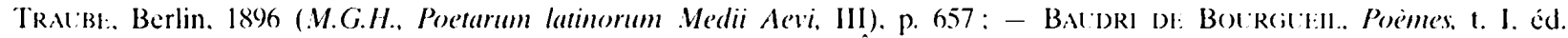
trad. Jean-Yves TIt.t.t:1Tt. Paris. 1998 (Auteurs latins du Moven Âge). p. 64. n 66 (super guidonem).

40. Die lateinische Dichter des deutschen Mittelatsers. V : Die Ottomenseit (.M.G.LI. Poctae Latini Medii Aevi. V). p. 317. n 6.

41. Gallia christiana. VIII. col. 1116-1117.

42. Histoire littéraire de la France. IV. Paris. 1866. p. 486: - Corpus des inscriptions de la France médiérale. t. 19. éd. R. Filrial: J. Michald, B. Mors. Paris. 1997, p. 86. 
On peut évidemment s'interroger sur la nature de cette épitaphe, texte effectivement inscrit sur le tombeau ou simple composition littéraire. Tout ce qu'on peut dire, c'est que les trois chroniqueurs qui la citent parlent expressément d'une inscription. Raoul de Dicet, mort en 1201 et donc contemporain d'Henri II, indique, avant de donner le texte le plus complet que l'on connaisse, qu'il s'agit d'une inscription qui orne le tombeau du roi (tumulum regis superscripti brevis exornat). Roger de Wendower $(\div 1285)$ et Matthieu Paris ( $\dagger$ 1259), parlent de "l'inscription du tombeau ». Épitaphe probablement inscrite sur le tombeau en 1189 donc, mais texte qui garderait son intérêt même s'il s'agissait d'une composition littéraire, comme témoin du retentissement qu'a dû avoir la brutale interruption d'une carrière exceptionnellement brillante.

L'épitaphe d'Henri II, conformément à ce qui se pratique au $\mathrm{XII}^{\mathrm{c}} \mathrm{s}$. ne développe pas une biographie : il a été roi, duc et comte, et a fait beaucoup de conquêtes. En ce sens, elle n'apprend rien à l'historien. C'est peut-être pour cette raison que les historiens d'Henri II s'en préoccupent peu. André Duchesne, dans son Histoire générale d'Angleterre (1614), César Égasse du Boulay dans son Historia universitatis Parisiensis... (1665) en donnent le texte, mais après eux on ne cite guère que le premier distique. L'épitaphe nous apprend pourtant qu'Henri II a été loué comme un nouvel Alexandre et que sa mort soudaine a eu un grand retentissement, tant il avait, sa vie durant, occupé le premier rang de la scène politique. On retrouve dans la Philippide de Guillaume le Breton en écho de ce choc : à quoi servent les honneurs, la gloire, l'argent... ? Du coup, à part une exhortation à faire le bien, il n'y a pas de note chrétienne, et en particulier on ne trouve pas de demande de prière, contrairement à ce qu'on a, en général, dans les épitaphes du $\mathrm{XII}^{*}$ s. Il faudrait resituer l'épitaphe d'Henri II dans la longue suite des épitaphes des rois et des grands princes, pour mieux en mesurer l'originalité. Mais ce serait une autre étude.

Il reste qu'en épigraphie il faut sans cesse faire jouer les séries, rechercher les sources littéraires, bibliques, liturgiques, hagiographiques, exégétiques, etc., et qu'en ce domaine on ne peut compter seulement sur la mémoire du déjà vu. Pour faciliter le travail de l'épigraphiste, j’ai donc entrepris depuis plusieurs mois la réalisation d'un fichier des formules, expressions, citations littéraires, bibliques. liturgiques, etc., pour les inscriptions du VII ${ }^{c}$ au XIII ${ }^{*}$ s. Dès que le logiciel, actuellement à l'étude, pour la saisie de ce fichier, sera opérationnel, ce travail. auquel devraient s'associer progressivement, d'après les premiers contacts pris, des collègues épigraphistes d'Allemagne, Espagne. Italie, pourra être accessible à tous les chercheurs. Il pourra aider à mieux comprendre la richesse des sources épigraphiques.

\author{
Robert FAVREAU \\ Centre d’Études Supérieures de Civilisation Médiévale \\ 24, rue de la Chaîne, BP 603 \\ $\mathrm{F}-86022$ POITIERS
}

